\title{
Face au mouvement de libéralisation des échanges : quelles politiques agricoles pour les PMA ? ${ }^{1}$
}

Michel PETIT

Institut agronomique méditerranéen, Montpellier

$<$ petitmichel@free.fr $>$

\begin{abstract}
In the poorest countries, agriculture is often the sector with the largest labour force and it provides the main source of incomes in rural areas wher the majority of poor people continue to live. Should these poor agricultures be protected from the rigour of competition on international markets where, in addition, prices are particularly unstable adding to food insecurity for those countries relying on those international markets to ensure a large proportion of their supplies? This question raises major controversies dominated by a priori ideological considerations. Yet rising to the world food challenge over the coming decades will undoubtedly require relying on domestic agriculture in poor countries because the economic growth of poor countries requires growth of their agriculture. Without it, poor countries would not have the financial resources necessary to fund massive food imports. And this calls for forceful public policies which go well beyond border protection, including the choice of public expenditures, particularly an emphasis on agricultural research, appropriate general economic policies to ensure favourable economic incentives to farmers.
\end{abstract}

Key words: agricultural policies, trade liberalization, least developped countries

zones rurales ? Faut-il alors protéger les agricultures peu productives des rigueurs des marchés internationaux, où par ailleurs les prix sont souvent très instables, ce qui contribue directement à l'insécurité alimentaire dans les pays qui auraient sacrifié leurs agricultures face à la concurrence internationale?

Ces questions, on le sait, soulèvent de vives controverses et des débats qui, hélas, ressemblent souvent à des dialogues de sourds. Les «Protectionnistes » soulignent la nécessité de protéger les agricultures pauvres du fait des grandes disparités entre les productivités moyennes du travail dans les agricultures paysannes pauvres et dans les agricultures modernes, différences qui devraient inéluctablement conduire à l'élimination des agricultures les plus pauvres. Ces arguments semblent irréfutables à première vue. Mais les «Libéraux » ont beau jeu de souligner que les interventions publiques sur les marchés sont sources de distorsions et d'inefficacités entraînant d'immenses gaspillages de ressources, surtout à long terme, et avec des conséquences négatives particulièrement graves pour les agriculteurs pauvres dans les pays pauvres. En outre, ces politiques impliquent souvent des coûts budgétaires, auxquels les États pauvres ne peuvent pas raisonnablement faire face, confrontés qu'ils sont à des besoins urgents de financements publics pour les infrastructures, la santé, l'éducation, etc. Ces positions, on le voit, se situent sur des plans différents : les arguments des uns ne répondent pas vraiment à ceux des autres. Et, trop souvent, on a l'impression que les prises de position dans ce débat sont essentiellement d'ordre idéologique. Comment alors progresser dans la réflexion pour véritablement éclairer les choix des acteurs publics et privés?

Le but de cette communication est de tenter de dépasser les querelles idéologiques sur ces questions importantes pour éclairer les choix de stratégies et de politiques à mettre en place pour faire face au défi alimentaire mondial au cours des prochaines décennies. Dans cette perspective, il apparaît clairement que les agricultures pauvres ne peuvent pas être ignorées. Leurs besoins requièrent des politiques publiques fortes, allant bien au-delà des protections aux frontières, protections dont on verra qu'elles ne jouent pas nécessairement un rôle central. Dans un premier temps, on verra comment l'examen du défi alimentaire mondial au cours des prochaines décennies amène à mettre l'accent sur la nécessité de politiques publiques vigoureuses pour soutenir le développement des agricultures dans les pays les moins avancés (PMA). On passera ensuite à une brève revue des principales dimensions de ces politiques publiques.

\section{Faire face au défi alimentaire mondial implique de fortes politiques publiques nationales dans les PMA}

Assurer une alimentation suffisante à une population mondiale croissante au cours des prochaines décennies requerra une croissance soutenue de la production agricole dans le monde. Les experts s'accordent sur un taux de $2 \%$ environ au cours des cinquante prochaines années. II ne s'agit là bien sûr que d'un ordre de 
grandeur ; entaché de beaucoup d'incertitudes car il dépend de plusieurs paramètres qui sont eux-mêmes incertains, surtout à une échéance de cinquante ans. Néanmoins, cet ordre de grandeur est suffisant pour les besoins de cet article.

Un taux de croissance annuel de $2 \%$ correspond à un triplement en 55 ans. Le défi que cette nécessité représente est considérable. En effet, tout d'abord, la croissance de la production agricole doit pour l'essentiel être réalisée dans les pays pauvres. L'idée que les pays riches pourraient assurer cette tâche doit être abandonnée. Un accroissement massif des volumes $\mathrm{d}^{\prime}$ aide alimentaire ne serait politiquement possible ni au nord ni au sud. Quant à un accroissement massif des importations de produits agricoles par les pays en développement, il impliquerait des besoins de financement tels que ces pays ne pourraient y faire face que par une forte croissance économique. Or, on touche là une contradiction fondamentale : il ne peut pas y avoir de forte croissance économique dans les pays les plus pauvres sans une forte croissance agricole, puisqu'une proportion importante de la population active (souvent plus de $50 \%$ ) est employée en agriculture. Aussi souhaitable qu'il soit, le transfert de main-d'œuvre de l'agriculture vers les autres secteurs économiques ne pourra pas être assez rapide pour assurer un emploi rémunérateur aux familles rurales et urbaines pauvres, et donc un revenu leur permettant d'acheter la nourriture dont elles auront besoin, au-delà de leur production propre pour ceux d'entre eux qui sont agriculteurs. Un scénario viable et vertueux de croissance et de développement qui serait fondé sur l'accroissement massif des importations agricoles et alimentaires est donc exclu.

II faudra par conséquent compter surtout sur l'accroissement de la production intérieure. Le niveau des performances passées en matière de croissance globale de la production agricole dans les pays en développement, supérieure à $2 \%$ par an depuis plusieurs décennies, peut être source d'optimisme à cet égard. Mais cette croissance n'a pas été uniforme et les PMA sont justement bien souvent ceux qui n'ont pas réussi dans la croissance de leurs agricultures ${ }^{2}$. Lorsqu'elle a eu lieu, cette croissance n'a été possible que grâce à la combinaison de trois facteurs : une croissance modérée des surfaces cultivées, une forte expansion de l'irrigation et la croissance des rendements par hectare. Pour les cinquante prochaines années, on ne pourra

\footnotetext{
$\overline{2}$ II est au demeurant intéressant de remarquer que des pays émergents comme I'Inde et la Chine, qui étaient parmi les plus pauvres il y a trente ou quarante ans, n'ont pu « décoller » que grâce à la croissance de leurs agricultures.
}

guère compter sur les deux premières sources de croissance : la mise en culture de nouvelles surfaces en quantités importantes sera freinée par des contraintes économiques et environnementales très difficiles à surmonter. Quant au développement de l'irrigation, il sera freiné par la nécessité dans de nombreux pays de transférer vers des usages non agricoles des volumes importants d'eau consacrés actuellement aux usages agricoles ${ }^{3}$. II ne reste donc que la croissance des rendements. Mais il faut être bien conscient que les institutions agronomiques, de recherche et d'enseignement supérieur, qui ont très souvent joué un rôle crucial dans les pays où la productivité agricole a progressé, sont généralement faibles dans les PMA. En outre, les approches qui ont permis les accroissements de rendement au cours des cinquante dernières années ne pourront pas suffire à l'avenir. Elles ont conduit dans les pays riches, et souvent aussi dans les pays du sud, à des pratiques qui ne sont pas durables car trop consommatrices d'énergie, trop polluantes et trop gourmandes en intrants chimiques divers. Ces conséquences négatives ont fondé un procès général de ce que l'on appelle le "productivisme » en agriculture, mot qui désigne la recherche des progrès de productivité de la terre et du travail avant tout, sans égard suffisant pour les conséquences sociales et environnementales négatives des pratiques mises en œuvre. On touche là à ce que les économistes appellent des « externalités", qui requièrent la mise en place de politiques publiques appropriées. La discussion de ces politiques sera maintenant l'objet de la deuxième partie de cet article.

\section{Quelles politiques publiques?}

La principale responsabilité pour assurer le développement des diverses agricultures des pays en développement, notamment pour les plus pauvres d'entre elles, devra être assumée par les gouvernements de ces pays. La communauté internationale, en particulier les autres gouvernements et les organisations internationales, a un rôle important à jouer mais qui ne peut que se situer en complément, en soutien, du rôle des gouvernements nationaux. Par ailleurs, les situations nationales étant très diverses, chaque pays doit adapter son action et ses politiques à sa situation spécifique, ce qui veut dire que les politiques publiques devront être très diverses. Tout discours général risque alors d'être faux ou au moins de ne pas être

\footnotetext{
${ }^{3}$ Une source paradoxale d'optimisme en la matière est le fait que dans de nombreux pays l'agriculture irriguée gaspille beaucoup d'eau, ce qui laisse une marge de manœuvre importante, et que l'on sait ce qu'il faudrait faire pour améliorer la situation [2].
}

pertinent. Néanmoins, quelques principes généraux restent pertinents et méritent d'être soulignés ici.

Depuis plus de dix ans, le consensus international sur ce qu'il convient de faire pour promouvoir le développement agricole et rural dans les pays en voie de développement s'est affirmé avec de plus en plus de force. II a été manifesté notamment au Sommet mondial de I'alimentation en 1996 et à la réunion du « Sommet mondial de l'alimentation plus cinq » tenue en juin 2002 à Rome. Certes, beaucoup ne voient dans les déclarations finales de ces grandes réunions internationales qu'un catalogue de vœux pieux et de promesses molles qui ne seront même pas tenues. Mais ces déclarations ont au moins une qualité : elles expriment un consensus international sur ce qu'il conviendrait de faire. Autrement dit, si le consensus est réel, le problème principal ne concerne plus le savoir, mais beaucoup plus le vouloir et le pouvoir. Qu'en est-il du développement agricole? Ayant moi-même dirigé la rédaction d'une stratégie sectorielle pour l'agriculture et le développement rural à la Banque mondiale en 1993, stratégie qui fut la première d'une série de documents de même nature rédigés par la Banque mondiale et par la plupart des autres agences de développement au cours des années qui suivirent, je suis convaincu qu'il y a effectivement un consensus international fort sur l'essentiel du contenu de ce que doivent être les politiques publiques en la matière. Un seul chapitre fait exception, celui du niveau souhaitable de la protection aux frontières et plus généralement celui des mérites de la libéralisation des échanges internationaux de produits agricoles.

Pour tenter de dépasser les prises de position purement idéologiques si fréquentes sur cette question, comme on l'a dit, il faut identifier précisément les points qui font l'objet de controverses et rechercher les informations empiriques pertinentes pour trancher. Certes, tous les points en débat ne peuvent pas être réglés de cette façon. Ainsi, seule l'expérience vécue pourrait permettre de juger en fin de compte si les risques impliqués par une éventuelle libéralisation des échanges sont aussi sévères que le disent ceux qui $s^{\prime} y$ opposent. Mais l'examen de quelques tendances passées montre en tout cas que la réalité est complexe et ne peut pas se réduire aux généralisations déduites des seules considérations idéologiques. Nous résumerons brièvement l'état du débat sur la nécessité de la protection des agricultures pauvres avant de revenir sur les autres dimensions des politiques nécessaires pour ces agricultures, dimensions qui à mes yeux sont beaucoup plus importantes pour leur avenir mais pourtant ne reçoivent pas I'attention qu'elles méritent. 
Quel danger la concurrence internationale représente-t-elle donc pour la production agricole intérieure? Pour répondre à cette question, on peut commencer par préciser les ordres de grandeur de quelques variables-clés. En effet, la pression de la concurrence internationale est souvent présentée comme irrésistible, risquant de conduire à la ruine des agricultures pauvres, à une augmentation massive des importations et, par là même, à un accroissement de la dépendance alimentaire. On constate effectivement que pour certains pays la part de la consommation totale couverte par la production intérieure, ce que l'on appelle le " coefficient d'autosuffisance», est devenue très faible. Tel est le cas par exemple de l'Algérie, où ce taux pour les céréales a oscillé autour de $25 \%$ au cours des années récentes, les céréales jouant dans ce pays comme dans beaucoup d'autres un rôle emblématique dans les débats sur cette question. Qu'en est-il de façon plus générale?

Avant d'examiner les faits, il faut souligner d'abord qu'une augmentation des importations ne serait pas nécessairement une catastrophe. Ainsi, peut-on remarquer que, parmi les pays méditerranéens, Malte, le Liban et le Portugal sont ceux qui, outre l'Algérie, ont les coefficients d'autosuffisance les plus faibles pour les céréales, respectivement 5,8\%, $11,3 \%$ et $33,9 \%$ en 2001 Ces exemples très divers illustrent que cet indicateur d'autosuffisance alimentaire n'est pas univoque. On devine bien en effet que pour ces trois pays, importer des quantités importantes de céréales, ce qui dénote certes un degré fort de dépendance alimentaire, $n^{\prime}$ est pas nécessairement une catastrophe et reflète peut-être au contraire un comportement tout à fait rationnel. Qu'en est-il des pays les plus pauvres? Constate-t-on une augmentation massive des importations? Quelles perspectives peut-on tracer pour l'avenir?

La catégorie des pays dits « pauvres et en déficit alimentaire » (Low Income Food Deficit Countries - LIFDC), proposée et utilisée par la FAO, est ici pertinente. On peut observer par exemple qu'entre 1962 et 2000 la production de céréales y a augmenté aussi rapidement que dans l'ensemble des pays en développement (en moyenne $3,0 \%$ et 2,9\% par an respectivement) et plus vite que dans les pays développés $(1,5 \%)$. Les importations de céréales se sont accrues à un taux plus faible que dans I'ensemble des pays en développement (3,5\% contre $4,5 \%$ ) et dans ces pays les plus pauvres le rapport Importations/Production intérieure n'est que de 0,10 en 2000, suggérant, pour I'ensemble de ces pays, une faible dépendance vis-à-vis des approvisionnements extérieurs pour cette catégorie importante de produits agricoles que sont les céréales. Les mêmes indi- cateurs calculés pour d'autres produits, comme les huiles végétales et la viande de poulet qui obéissent à des dynamiques d'offre et de demande assez différentes, confirment ce diagnostic général. Par ailleurs, les principales projections disponibles sur les prochaines décennies montrent que la dépendance vis-à-vis des sources extérieures d'approvisionnement n'est pas appelée à changer radicalement. Mais elle risque fort, il est vrai, d'augmenter significativement pour certains produits et surtout pour certains pays. Rien $n$ 'indique cependant que les prévisions les plus pessimistes des opposants de la libéralisation des échanges quant à l'élimination de pans entiers de la paysannerie dans les pays pauvres sous l'effet de la concurrence des pays riches soit en train de se réaliser. Bien sûr, ces données empiriques ne permettent pas de trancher définitivement le débat. Elles montrent à tout le moins que les réalités sont complexes et ne peuvent pas être réduites à des slogans simplistes.

Parmi les autres dimensions des politiques publiques affectant l'agriculture, le point le plus important porte probablement sur le montant et le choix des dépenses publiques. Le rôle nécessaire de l'État dans le développement des infrastructures publiques et l'importance de ces infrastructures pour le développement agricole sont unanimement reconnus. Mais les ressources fiscales des pays les plus pauvres sont très limitées, alors que les besoins sont immenses. Le sous-équipement en infrastructures est donc la norme habituelle, à laquelle tout un chacun tend malheureusement à se résigner trop facilement. II en est souvent de même pour les services publics dans les domaines de l'éducation et de la santé. Par suite, les débats publics sur la répartition des dépenses publiques sont souvent ternes, alors qu'il y aurait pourtant beaucoup à dire pour dénoncer les dépenses excessives sur d'autres chapitres (défense, sécurité, dépenses de prestige, etc.).

La recherche agronomique est I'un des secteurs fréquemment mal traités dans l'établissement du budget de l'État. Les nombreux travaux économétriques montrant une très grande rentabilité des dépenses de recherche sont tellement nombreux et convergents que l'on peut considérer qu'ils démontrent, malgré leurs limites évidentes, que la plupart des gouvernements investissent insuffisamment dans ce secteur. Mais au-delà de ces aspects financiers, d'autres réformes profondes sont souvent nécessaires dans les politiques de recherche pour l'agriculture (encadré 1).

La recherche, comme on l'a dit, est en effet appelée à jouer un rôle stratégique face au défi alimentaire mondial. Le défi à relever n'est pas simple car il faut à la fois fournir une alimentation à bon marché à ceux qui sont des acheteurs nets de nourriture et assurer un revenu aussi élevé que possible à ceux qui tirent l'essentiel de leurs revenus, en argent et en nature, de l'exercice d'une activité agricole dans les zones défavorisées et qui ont intérêt à ce que les prix agricoles soient aussi élevés que possible. Gérer cette contradiction d'intérêts n'est pas du ressort de la recherche agronomique; mais le défi qu'elle doit relever est de trouver des solutions techniques permettant d'accroître la productivité du travail dans des situations agricoles difficiles, ce qui n'est jamais simple!

On a dit ci-dessus que les approches passées ne sont plus appropriées pour les cinquante prochaines années parce qu'elles ont conduit à des pratiques qui ne sont pas durables. Ceci implique un autre ensemble de défis scientifiques pour la recherche agronomique. Elle ne pourra les relever qu'en s'ouvrant davantage à l'écologie et en intégrant mieux les apports des sciences sociales dans le cadre d'approches systémiques qui sont seules susceptibles de prendre en compte l'immense complexité des situations agricoles très diverses dans les PMA.

Les défis scientifiques et organisationnels qui viennent d'être évoqués sont particulièrement aigus dans de nombreux systèmes nationaux de recherche agronomique (SNRA) des pays les plus pauvres. Dans bien des cas, particulièrement en Afrique, les dysfonctionnements institutionnels graves sont la règle plutôt que I'exception. II n'est pas surprenant alors que la productivité des chercheurs soit faible, que les besoins des agriculteurs ne soient pas entendus et que la liaison avec la formation laisse beaucoup à désirer. Porter remède à ces dysfonctionnements doit être au cœur de toute politique de recherche. II y faut beaucoup de volonté politique et de constance de la part des pouvoirs publics. Heureusement, certains exemples, ceux du Brésil, de l'Inde et de I'Egypte notamment, montrent que lorsque ces conditions sont réunies, les progrès peuvent être spectaculaires.

Plus généralement, les politiques économiques qui influencent les termes de l'échange pour les agriculteurs sont aussi très importantes. Rares sont les exemples de développement agricole réussi lorsque les incitations économiques auxquelles les agriculteurs faisaient face étaient très défavorables. Or on sait que, contrairement aux pays développés qui le plus souvent soutiennent leurs agricultures, les pays en développement tendent à «l'exploiter ». Ainsi, on a pu montrer que les principales causes de «l'exploitation » des agriculteurs dans les pays en voie de développement sont la surévaluation du taux de change et la protection des industries, alors que dans de nombreux pays les exportations de produits agricoles sont soumises à une taxation [3]. Les agriculteurs sont les principales victimes de ces distorsions écono- 


\section{Encadré 1}

La recherche agronomique est l'un des secteurs fréquemment mal traités dans l'établissement du budget de l'État (Pardey P. et N. Beintema, 2001).

Les nombreux travaux économétriques montrant une très grande rentabilité des dépenses de recherche sont tellement nombreux et convergents que l'on peut considérer qu'ils démontrent, malgré leurs limites évidentes, que la plupart des gouvernements investissent insuffisamment dans ce secteur. Mais au-delà de ces aspects financiers, d'autres réformes profondes sont souvent nécessaires dans les politiques de recherche pour l'agriculture.

La recherche est en effet appelée à jouer un rôle stratégique face au défi alimentaire mondial. Le défi à relever n'est pas simple car il faut à la fois fournir une alimentation à bon marché à ceux qui sont des acheteurs nets de nourriture et assurer un revenu aussi élevé que possible à ceux qui tirent l'essentiel de leurs revenus, en argent et en nature, de l'exercice d'une activité agricole dans les zones défavorisées et qui ont intérêt à ce que les prix agricoles soient aussi élevés que possible. La première catégorie est la plus nombreuse, elle inclut les résidents urbains, y compris les plus pauvres d'entre eux, mais aussi les plus pauvres parmi les ruraux qui sont le plus souvent acheteurs nets de nourriture et, à ce titre, bénéficiaires de prix agricoles bas, point essentiel qui semble avoir échappé à beaucoup de nos collègues français !

Mais il est vrai que de nombreux agriculteurs travaillent dans des conditions difficiles et auraient au contraire intérêt à ce que les prix agricoles soient élevés. C'est bien leurs situations que les défenseurs du protectionnisme mettent en avant.

Cette question du protectionnisme sera discutée ci-dessous. Pour ce qui concerne la recherche agronomique, le défi est de trouver des solutions techniques permettant d'accroître la productivité du travail dans des situations agricoles difficiles, ce qui n'est jamais simple!

On a dit ci-dessus que les approches passées ne sont plus appropriées pour les cinquante prochaines années parce qu'elles ont conduit à des pratiques qui ne sont pas durables, car trop consommatrices d'énergie, trop polluantes et trop gourmandes en intrants chimiques divers. Ceci implique que la recherche agronomique, partout dans le monde, fait face à des défis scientifiques importants. Trop souvent, « le niveau écologique reste délaissé à un moment où les applications de cette discipline au monde agronomique et à la gestion des systèmes et des ressources naturelles explosent (émergence de l'agroécologie, de l'ingénierie des systèmes écologiques, des dispositifs de terrain adaptés à ces études). L'apport des sciences sociales n'est pas assez intégré. Malgré les affichages l'interdisciplinarité reste à construire. L'étude intégrée des agrosystèmes reste encore largement à faire et les solutions techniques à trouver. »

Ces critiques, qui font l'objet d'un vaste consensus, s'appliquent peu ou prou à l'ensemble des institutions de recherche agronomique dans le monde. La direction générale de ce qu'il faut faire pour dépasser ces limites est pourtant claire : la recherche agronomique doit s'ouvrir davantage aux disciplines biologiques (biologie moléculaire, biologie intégrative, dépasser le modèle de la boîte noire où l'on s'intéresse seulement aux intrants, et aux produits sans s'intéresser aux mécanismes internes).

La recherche agronomique doit s'ouvrir plus particulièrement à l'écologie et à la modélisation des systèmes complexes; elle doit développer les pratiques pluridisciplinaires, voire transdisciplinaires, pour utiliser la terminologie très pertinente ici de Piaget, et engager des recherches sur les systèmes d'acteurs avec lesquels elle travaille pour transformer les agricultures. Mais il faut bien convenir que ces prescriptions sont plus faciles à formuler qu'à mettre en œuvre. Elles appellent des partenariats nouveaux ou élargis plus approfondis et plus réels, que ce que I'on rencontre le plus souvent sur le terrain.

Les défis scientifiques et organisationnels qui viennent d'être évoqués sont particulièrement aigus dans de nombreux systèmes nationaux de recherche agronomique (SNRA) des pays du sud. Dans bien des cas, particulièrement en Afrique, les dysfonctionnements institutionnels graves sont la règle plutôt que l'exception : les installations et les équipements sont mal entretenus ou ne le sont pas du tout. Les salaires sont versés avec retard, l'approvisionnement en consommables est problématique et les frais de mission sont difficiles à se faire rembourser. II n'est pas surprenant alors que la productivité des chercheurs soit faible, que les besoins des agriculteurs ne soient pas entendus et que la liaison avec la formation laisse beaucoup à désirer. Porter remède à ces dysfonctionnements doit être au cœur de toute politique de recherche. II y faut beaucoup de volonté politique et de constance de la part des pouvoirs publics. Heureusement, certains exemples, ceux du Brésil, de l'Inde et de l'Egypte notamment, montrent que lorsque ces conditions sont réunies, les progrès peuvent être spectaculaires.

En outre, il ne serait pas raisonnable que tous les SNRA, ceux des très gros pays, comme l'Inde, la Chine ou le Brésil par exemple, et ceux des plus petits, adoptent la même stratégie. Tous n'ont pas les mêmes capacités. Les questions du dimensionnement optimal de chaque système national, de l'éventail de disciplines à couvrir et des partenariats à développer dans des coopérations bilatérales, régionales et internationales se posent. Pourtant de nombreux pays, petits et moyens, ont beaucoup de mal à y faire face, y compris même à formuler les questions de ce type. miques, qui sont souvent de grande ampleur. Tous ceux qui veulent défendre les intérêts des plus pauvres devraient donc les dénoncer avec force.

Enfin, il faut souligner que les plus pauvres, en particulier les agriculteurs pauvres, sont très dépendants des ressources naturelles : sols, eau et biodiversité en particulier. Dans de nombreux pays pauvres, l'État n'assure pas ou très mal la bonne gestion des ressources communes (forêts, pâturages, eaux, etc.) qui jouent pourtant un très grand rôle dans la vie et la subsistance des plus pauvres. Améliorer les politiques publiques dans ce domaine est donc bien souvent de la plus grande importance, même si $c^{\prime}$ est souvent politiquement difficile [2].

\section{Conclusion}

La mondialisation et la libéralisation des échanges qui l'accompagne n'impliquent pas une réduction massive du rôle de la puissance publique. Ce mouvement requiert au contraire une défense beaucoup plus vigoureuse que ce n'est souvent le cas des intérêts des plus pauvres, qui sont souvent en situation de faiblesse politique. Ceci est particulièrement vrai pour l'agriculture et les agriculteurs dans les pays les plus pauvres. De fortes politiques publiques sont nécessaires ${ }^{4}$. Trop souvent cependant les débats sur ces questions dans les pays francophones se limitent à la nécessité de protections aux frontières. Or, sur ce point, l'examen des données empiriques pertinentes et disponibles révèle une réalité complexe, qu'il est dangereux de réduire à quelques généralités simplistes. De plus, limiter la discussion des mérites respectifs du protectionnisme et de la libéralisation des échanges a le grand danger d'occulter des conclusions robustes sur d'autres dimensions fort importantes des politiques publiques de soutien aux agricultures paurres,

\footnotetext{
${ }^{4}$ Cette affirmation pose question: Comment faire valoir cette nécessité ? Dans quel cadre ou devant quelles instances la défendre dans la mesure où, semble-t-il, les agences internationales fondent leurs interventions sur un contournement par le marché, de l'action publique ? Tout d'abord, cette perception est probablement erronée : I'examen des stratégies de soutien au développement rural proposées par les agences d'aide au développement, à commencer par celles de la Banque mondiale, ne prônent pas du tout un retrait pur et simple de la puissance publique. Plus fondamentalement, ce qui est en cause ici c'est I'idéologie libérale qui fonde trop souvent le discours dominant des responsables de ces organisations alors que leurs pratiques sont heureusement beaucoup plus pragmatiques dans la plupart des cas.
} 
comme le choix des dépenses publiques, la nécessité et la difficulté de promouvoir une recherche agronomique dynamique, la nécessité de corriger les distorsions de politiques économiques dont souffrent de nombreux agriculteurs, y compris les plus pauvres d'entre eux, et la nécessité de politiques de gestion des ressources naturelles plus efficaces et tenant mieux compte des enjeux pour les plus pauvres $d^{\prime}$ une meilleure gestion de ces ressources.

\section{RÉFÉRENCES}

1. PETIT M. Le développement des agricultures face a l'accroissement des besoins locaux dans un contexte de mondialisation. In : Enjeux et Perpectives de la recherche agronomique dans les pays en développement. Les Colloque de l'Académie d'agriculture. № 1, 2004 : 101-12.

2. PETIT M. Développement durable à l'échelle de la planète et gestion des ressources en eau et en sols. ». Géoscience 2003 ; 335 : 6-7 ; (Compte
Rendu du colloque : «Effet de serre, impact et solutions : quelle crédibilité ? ", Académies des Sciences, de I'Agriculture et des Technologies, Paris, septembre 2002).

3. KRUEGER AO, SCHIFF M, VALDES A. Agricultural Incentives in Developing Countries: Measuring Effects of Sectorial and Economy-wide Policies. World Bank Econ Rev $1998 ; 2$ : 255-71. 